Auslegungsgründsätze deutlich werden. Angesichts der gefestigten Rechtsprechung zu Art. 6 CISG vermag das Urteil des Cour de Cassation nur zu verwirren und kann daher bei der Auslegung des Art. 6 CISG durch die Gerichte der übrigen Vertragsstaaten, die sich mit Auslegungsfragen befassen, höchstens als Negativbeispiel Beachtung finden, zumal die Gerichte der Vertragsstaaten das CISG immer als nationales und nicht etwa als ausländisches Recht anzuwenden haben, so dass seine Anwendung auch in der Revisionsinstanz in vollem Umfang nachprüf- bar ist. ${ }^{77}$ Es bleibt daher zu wünschen, dass die hier besprochene Entscheidung vereinzelt bleibt und weder bei den französischen Instanzgerichten noch bei den Gerichten anderer Vertragstaaten Gefolgschaft findet.

\footnotetext{
77 Schlechtriem/Ferrari, Art. 1 CISG, Rn. 76.
}

\title{
Die Vertragswidrigkeit im Rahmen des Art. 82 Abs. 2 lit. c CISG
}

\begin{abstract}
Wiss. Assistent Florian Mohs, Basel
Sachmängel, Schutzrechtsmängel und allgemeine Rechtsmängel werden in den Tatbeständen der Rechtsbehelfe auf der Käuferseite unterschiedlich behandelt. Im Schrifttum werden zu deren Verhältnis zueinander verschiedene Auffassungen vertreten. Dieser für die Praxis wichtigen Frage geht die folgende Untersuchung nach.
\end{abstract}

\section{Der Regelungszusammenhang des Art. 82 Abs. 2 lit. c CISG}

Unter der Überschrift „Wirkungen der Aufhebung“ ist in den Art. 81-84 CISG die Rückabwicklung von Verträgen geregelt. Dort sind die Folgen der Vertragsaufhebung für beide Parteien einheitlich festgelegt, bildlich gesprochen hinter die Klammer gezogen, während die Voraussetzungen der Vertragsaufhebung in Art. 49 CISG für den Käufer und in Art. 64 CISG für den Verkäufer getrennt normiert sind.

Art. 82 Abs. 1 CISG normiert eine Vertragsaufhebungssperre $^{1}$ für den Käufer ${ }^{2}$, d.h. sein Vorliegen hindert die Ausübung dieses Rechtsbehelfs und entspricht damit funktional den $\S \S 350-353$ BGB a. F. ${ }^{3}$ So verliert der Käufer das Recht zur Vertragsaufhebung bzw. zur Ersatzlieferung ${ }^{4}$, wenn es ihm unmöglich ${ }^{5}$ ist, er also tatsächlich daran gehindert ist, die Ware im wesentlichen ${ }^{6}$ so zurückzugeben, wie er sie erhalten hat.

Art. 82 Abs. 2 CISG enthält etliche Ausnahmen zu dieser

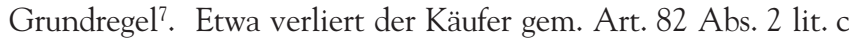
CISG das Recht zur Vertragsaufhebung nicht, wenn er die Ware im normalen Geschäftsverkehr weiterverkauft. Allerdings kann sich der Käufer dann nicht auf diese Ausnahmeregelung berufen, wenn er die Ware weiterverkauft hat, obwohl er die Vertragswidrigkeit ${ }^{8}$ bereits entdeckt hatte oder hätte entdeckt haben müssen.

Die hier bedeutsam werdende Fragestellung, ob der Begriff der Vertragswidrigkeit alle Fälle der Schlechtlieferung umfasst, oder nur Sachmängel, also nicht Rechtsmängel meint, ist Gegenstand dieses Beitrags. Im Zusammenhang mit Art. 82 CISG ist diese Frage in der Literatur bisher nicht behandelt worden, obwohl sie an anderen Gesetzesstellen ${ }^{9}$ stark umstritten ist. Dies gilt insbesondere für die einschränkende Sonderregelung der Nacherfüllung in Art. 46 Abs. 2 , 3 CISG und für das Recht zur Minderung des Kaufpreises gem. Art. 50 CISG.

\section{Meinungsstand zu Art. 46 Abs. 2, 3, Art. 50 CISG}

Zur Frage, ob der Käufer im Falle eines Rechtsmangels uneingeschränkt Ersatzlieferung oder Nachbesserung verlangen bzw. den Kaufpreis mindern kann, werden in der Literatur unterschiedliche Auffassungen vertreten, die im Nachfolgenden diskutiert werden sollen.

1 Vgl. Schlechtriem, Restitution und Bereicherungsausgleich in Europa, Eine rechtsvergleichende Darstellung, Band 1, 2000, Kapitel 3, Rn. 8 (S. 409), 576 (S. 696).

${ }^{2}$ Gesetzestechnisch gesehen wurde diese Aufhebungssperre in doppelter Hinsicht systematisch falsch eingeordnet. Erstens befindet sie sich in Kapitel V. „Gemeinsame Bestimmungen über die Pflichten des Verkäufers und des Käufers“, obwohl sie ausschließlich das Aufhebungsrecht des Käufers sperrt. Zweitens wird sie im Zusammenhang des Abschnitts V. „Wirkungen der Aufhebung" behandelt, obwohl sie eine Voraussetzung normiert, vgl. Krebs, Die Rückabwicklung im UN-Kaufrecht, 2000, S. 92, Fn. 569.

3 So die bis 31. 12. 2001 geltende Fassung des BGB. Auch wenn durch die Modernisierung des internen Schuldrechts die $§ \S 350-353$ BGB gestrichen wurden, ist die hier behandelte Problematik unverändert aktuell, da das UN-Kaufrecht als Sonderrecht für grenzüberschreitende Warenkäufe von dem Schuldrechtsmodernisierungsgesetz unberührt blieb.

${ }^{4}$ Da sowohl die Vertragsaufhebung als auch die Ersatzlieferung zur Rückabwicklung von bereits Geleistetem führt, hat Art. 82 CISG beide Sachverhalte parallel geregelt. Wenn im Nachfolgenden nur von Vertragsaufhebung gesprochen wird, so gilt das entsprechend auch für den Fall der Ersatzlieferung.

${ }^{5}$ Unmöglichkeit in diesem Zusammenhang meint nicht die Begrifflichkeit des deutschen internen Rechts der Leistungsstörungen (vgl. Art. 7 CISG), Schlechtriem/Leser/Hornung, Kommentar zum Einheitlichen UNKaufrecht - CISG -, 3. Aufl., 2000, Art. 82, Rn. 9.

6 Das Merkmal Wesentlichkeit inkorporiert eine Bagatellreglung, die verhindert, dass unwesentliche Veränderungen dem Aufhebungsrecht schaden, Schlechtriem/Leser/Hornung, a.a.O. (Fn. 5), Art. 82, Rn. 11.

7 Die Ausnahmen greifen derart weit, dass der eigentlichen Grundregel kaum noch Anwendungsbereich bleibt, und sich das Verhältnis Regel - Ausnahme umzudrehen scheint, Schlechtriem/Leser/Hornung, a.a.O. (Fn. 5), Vor Artt. 81-84, Rn. 12; Art. 82, Rn. 12.

8 Engl.: „lack of conformity“; franz.: „défaut de conformité“.

9 Der Begriff der Vertragswidrigkeit findet sich im Übereinkommen in den Art. 35 Abs. 3, Art. 36, 37, 39, 40, 46 Abs. 2, 3, Art. 50, 51, 82 Abs. 2 lit. c CISG. 


\section{Gleichlauflösung de lege lata ausgeschlossen}

Eine Meinung in der Literatur negiert den Unterschied zwischen Sach- und Rechtsmängeln auf der Rechtsbehelfsebene gänzlich $^{10}$. Dem Käufer stehe auch bei einem Rechtsmangel immer das Recht zur Minderung nach Art. 50 CISG zu ${ }^{11}$. Dafür könne er aber stets nur unter den einschränkenden Voraussetzungen des Art. 46 Abs. 2, 3 CISG Ersatzlieferung bzw. Nachbesserung verlangen ${ }^{12}$.

Richtig ist, dass das Leistungsstörungsrecht des CISG auf dem einheitlichen Begriff der Pflichtverletzung ${ }^{13}$ aufbaut, vgl. Art. 45, $61 \mathrm{CISG}^{14}$. Allerdings verweisen diese Anknüpfungsnormen in ihren Unterabsätzen a) auf die Tatbestände der einzelnen Rechtsbehelfe. Ihnen kommt deshalb lediglich eine ordnende Funktion $\mathrm{zu}^{15}$.

Die in den Tatbeständen der Art. 46 Abs. 2, 3, Art. 50 CISG benutzte Formulierung „Ist die Ware nicht vertragsgemä $\beta,[\ldots]$ ]“ spricht dafür, dass sie das Vorliegen eines Sachmangels voraussetzen, also nicht für Rechtsmängel gelten. Diese Auffassung wird vom Wortlaut der Überschrift zu Teil III., Kapitel II., Abschnitt II. „Vertragsmäßigkeit der Ware sowie Rechte oder Ansprüche Dritter" getragen, die zwischen Vertragsgemäßheit einerseits und Rechtsmängeln andererseits differenziert ${ }^{16}$.

Auch wenn ein völliges Gleichlaufen von Sach- und Rechtsmängeln auf der Rechtsbehelfsebene ${ }^{17}$, eine praktische Kongruenz der Rechtsbehelfe, rechtspolitisch wünschenswert erscheint $^{18}$, so ist sie doch de lege lata ausgeschlossen ${ }^{19}$.

\section{Die allgemeine Auffassung im Zusammenhang mit Art. 46 Abs. 2, 3 CISG}

In der Literatur wird allgemein vertreten, dass die einschränkende Sonderregelung des Art. 46 Abs. 2, 3 CISG nur im Fall von Sachmängeln greife, der Käufer rechtsmangelbehafteter Ware also stets uneingeschränkt Ersatzlieferung und Nachbesserung verlangen könne ${ }^{20}$. Für diese Auffassung streitet das systematische Argument, dass sich Art. 46 Abs. 2, 3 CISG bei der Festlegung der Frist, innerhalb derer der Käufer Nacherfüllung verlangt haben muss, nur auf die für Sachmängel geltende Anzeige nach Art. 39 CISG, nicht aber auf die für Rechtsmängel geltende Anzeige nach Art. 43 CISG bezieht ${ }^{21}$.

${ }^{10}$ Herber/Czerwenka, Internationales Kaufrecht: Kommentar zu dem Übereinkommen der Vereinten Nationen vom 11. April 1980 über Verträge über den internationalen Warenkauf, 1991, Art. 45, Rn. 2; Wolff, Die Rechtsmängelhaftung nach dem Uniform Commercial Code und dem UNKaufrecht, 1990, S. 164, derzufolge der Begriff der „conformity“ auf Rechtsbehelfsebene (Kapitel II., Abschnitt III.) auch Rechtsmängel erfasse, hingegen auf der Ebene der Haftungsstandards (Kapitel II., Abschnitt II.) nur Sachmängel regele.

${ }^{11}$ Herber/Czerwenka, a.a.O. (Fn. 10), Art. 50, Rn. 3; Reinhart, UNKaufrecht, 1991, Art. 50, Rn. 2; Wolff, a.a.O. (Fn. 10), S. 173 f.

${ }^{12}$ Herber/Czerwenka, a.a.O. (Fn. 10), Art. 46, Rn. 6, 9; Reinhart, a.a.O. (Fn. 11), Art. 46, Rn. 3f.; Wolff, a.a.O. (Fn. 10), S. 163f., 166.

13 Das Gesetz selbst spricht zwar von Vertragsbruch oder von der Nichterfüllung bestehender Pflichten. Schlechtriem zufolge bezeichnet aber der Begriff der Pflichtverletzung die einheitliche Störungsform präziser, da auch im CISG das Verhalten und die Leistung des Schuldners an einem durch Vertrag und Gesetz bestimmten Pflichtenprogramm zu messen seien, Schlechtriem, Der Einfluß des UN-Kaufrechts auf die Entwicklung des deutschen und des internationalen Schuldrechts, IHR 2001, S. 16.
${ }^{14}$ Der einheitliche Begriff der Pflichtverletzung entstand durch das Bedürfnis, die verschiedenen Rechtsbehelfe in einen einheitlichen Satz von Rechtsbehelfen zusammenzufassen, der sprachlich alle Leistungsstörungen, insbesondere auch die Rechtsmängel, einbeziehen musste. Dieses Bedürfnis ist in YB IV, S. 52, Art. 41, Nr. 115 (in: Honnold, Documentary History of the Uniform Law for International Sales, 1989, S. 129) artikuliert und in YB IV, S. 59, Art. 41, Nr. 163 (in: Honnold, a.a.O., S. 136) umgesetzt worden.

15 Schlechtriem/Huber, a.a.O. (Fn. 5), Art. 45, Rn. 1; Schlechtriem/Hager, a.a.O. (Fn. 5), Art. 61, Rn. 1, 3; Hirner, Der Rechtsbehelf der Minderung nach dem UN-Kaufrecht (CISG), 2000, S. 199, 203. Im Gegensatz dazu normieren Art. 45 Abs. 1 lit. b CISG und Art. 61 Abs. 1 lit. b CISG die schadensersatzrechtlichen Anspruchsgrundlagen, da in Art. 74ff. CISG nur noch der Umfang der Ersatzpflicht bestimmt wird, Schlechtriem/Huber, a.a.O. (Fn. 5), Art. 45, Rn. 1.

${ }^{16}$ Schlechtriem/Schwenzer, a.a.O. (Fn. 5), Art. 41, Rn. 20; Langenecker, UN-Einheitskaufrecht und Immaterialgüterrechte, Die Rechtsmängelhaftung bei internationalen Kaufverträgen nach dem UN-Kaufrechtsabkommen unter besonderer Berücksichtigung von Immaterialgüterrechten, 1993, S. 52, 95, 263; a.A. Schlechtriem/Schlechtriem, a.a.O. (Fn. 5), Art. 3 VertragsG, Rn. 5, demzufolge Rechtsmängel an sich unter den Begriff „Vertragswidrige Beschaffenheit" fallen, da sie im gleichen Abschnitt wie Sachmängel unter „Vertragsmäßigkeit der Ware“ geregelt seien. Dabei wird m.E. dem zweiten Teil der Überschrift „sowie Rechte und Ansprüche Dritter“ zu wenig Bedeutung zugemessen. Die Überschrift wurde bei den Verhandlungen geändert, um auch Rechtsmängel zu erfassen, YB VIII, S. 41, Art. 25, Nr. 225 (in: Honnold, a.a.O. (Fn. 14), S. 334); so auch Prager, Verkäuferhaftung und ausländische gewerbliche Schutzrechte, Die Haftung des Verkäufers beweglicher Sachen für deren Freiheit von gewerblichen Schutzrechten oder Urheberrechten nach dem UN-Kaufrechtsübereinkommen vom 11. April 1980, 1987, S. 135, 136, 203.

17 Auswirkungen der Differenzierung in Sach-, Schutzrechts- und allgemeine Rechtsmängel fänden sich dann nur noch in folgenden Punkten: Zweijährige Ausschlussfrist nur bei Sachmängeln gem. Art. 39 Abs. 2 CISG; verschieden hohe Anforderungen an die Haftung bzw. etwaige Haftungsausschlüsse, vgl. Art. 35, 41, 42 CISG. Aufgrund dieser Unterschiede würde die schwierige und bisweilen missliche Abgrenzung zwischen diesen Leistungsstörungen, insbesondere zwischen Sachmängeln und allgemeinen Rechtsmängeln, z.B. im Fall öffentlich-rechtlicher Belastungen, auch bei Gleichlauf der Rechtsbehelfe bestehen bleiben.

18 Schlechtriem, Internationales UN-Kaufrecht, 1996, Rn. 202, im Zusammenhang mit dem Minderungsrecht.

${ }^{19}$ Schlechtriem/Huber, a.a.O. (Fn. 5), Art. 50, Rn. 8, demzufolge eine Einheitslösung de lege ferenda gewiss denkbar gewesen wäre.

${ }^{20}$ Staudinger/Magnus, Kommentar zum Bürgerlichen Gesetzbuch mit Einführungsgesetz und Nebengesetzen, Wiener UN-Kaufrecht (CISG), 1999, Art. 46, Rn. 17; Art. 41, Rn. 23, der auf die fehlende Erwähnung von Art. 43 CISG in Art. 46 Abs. 2, 3 CISG abstellt und behauptet, dass i.d.R. unwesentliche Rechtsmängel den Gebrauch oder den Absatz unmöglich machen. Das überzeugt nicht, soweit es um Art. 42 CISG geht, da schutzrechtbehaftete Ware prinzipiell weiterverkauft werden kann, Soergel/ Lüderitz/Schüßler-Langeheine, Bürgerliches Gesetzbuch, Bd. 13, Übereinkommen der Vereinten Nationen über Verträge über den internationalen Warenkauf, 2000, Art. 46, Rn. 13; Achilles, Kommentar zum UN-Kaufrechtsübereinkommen (CISG), 2000, Art. 46, Rn. 2; Langenecker, a.a.O. (Fn. 16), S. 268, 269; Hirner, a.a.O. (Fn. 15), S. 197ff., 200f., 204; a.A. Enderlein, in: International Sale of Goods, Dubrovnik Lectures, 1986, S. 190; Enderlein/ Maskow/Strohbach, Internationales Kaufrecht, 1991, Art. 46, Nr. 3.

21 Schlechtriem/Schwenzer, a.a.O. (Fn. 5), Art. 41, Rn. 20.

22 Unter Geltung des BGB hätte man kaum Probleme, die mit einem Rechtsmangel behaftete Ware als nicht vertragsgemäß zu bezeichnen. Ebenso unter Geltung des UCC, vgl. § 2-106(2): „Goods [...] are conforming or conform to the contract when they are in accordance with the obligations 


\section{Minderung wegen Rechtsmangels gem. Art. 50 CISG?}

\section{a) Einheitliche Auslegung}

Ausgehend von Wortlaut und Systematik der Konvention und entgegen dem allgemeinen Sprachgebrauch ${ }^{22}$ wird die Auffassung vertreten, dass der Begriff der Vertragswidrigkeit technisch so zu verstehen sei, dass er nur Sachmängel umfasse. Dieser terminus technicus des Übereinkommens müsse als solcher an den verschiedenen Gesetzesstellen in gleicher Weise verstanden werden $^{23}$. Deshalb finde Art. 50 CISG nur im Fall eines Sachmangels Anwendung ${ }^{24}$.

Diese einheitliche Auslegung fußt auf der Entstehungsgeschichte und damit auf dem Willen des historischen Gesetzgebers, demzufolge Art. 46 Abs. 2, 3, Art. 50 CISG nur auf die Sachmängelhaftung angewendet werden sollten ${ }^{25}$.

Schließlich kann dieser Auffassung aufgrund ihrer Einheitlichkeit und Klarheit eine gewisse Rechtssicherheit nicht abgesprochen werden ${ }^{26}$.

\section{b) Ergebnisorientierte Auslegung}

Von dem freilich verständlichen Bestreben geleitet, auch dem Käufer rechtsmangelbehafteter Ware das Recht zur Minderung zu gewähren ${ }^{27}$, wird die Auffassung vertreten, dass der Anwendungsbereich der fraglichen Vorschriften autonom bestimmt werden müsse. Als systematisches Argument wird die Regelung des Art. 44 CISG angeführt. Im Falle einer vernünftigen Entschuldigung könne der Käufer, der die nach Art. 39 CISG oder Art. 43 CISG notwendige Anzeige unterlassen hat, trotzdem sein Minderungsrecht nach Art. 50 CISG ausüben ${ }^{28}$. Es sei nicht einzusehen, warum ein Käufer nur bei unterlassener und nicht auch bei ordnungsgemäßer Rüge zur Minderung berechtigt sein solle ${ }^{29}$.

Dieses Gegenargument ist aber weder zwingend ${ }^{30}$ noch überzeugend $^{31}$, da Art. 44 CISG im Einklang mit seinem Wortlaut und entsprechend seinem Sinn, über eine unterlassene Anzeige hinwegzuhelfen, nur im Sinne einer Rechtsgrundverweisung verstanden werden kann. Demnach besteht das Recht zur Minderung lediglich dann, wenn alle sonstigen Voraussetzungen zur Begründung des Gestaltungsrechts gegeben sind ${ }^{32}$. Dies ist aber bei einem Rechtsmangel gerade nicht der Fall ${ }^{33}$. Außerdem steht der Wortlaut des Art. 44 CISG einer Auslegung dahingehend, dass dem Käufer das Recht zur Minderung nur im Falle einer unterlassenen Anzeige nach Art. 39 CISG zusteht, er im Falle von Art. 43 CISG aber auf Schadensersatz zu verweisen ist, nicht entgegen.

under the contract." A.A. Prager, a.a.O. (Fn. 16), S. 203 m.w.N., demzufolge auch der normale Sprachgebrauch nur Sachmängel erfasst; so auch Langenecker, a.a.O. (Fn. 16), S. 264.

23 Schlechtriem/Huber, a.a.O. (Fn. 5), Art. 46, Rn. 24, 27, 57; Art. 50, Rn. 8; Karollus, UN-Kaufrecht: Eine systematische Darstellung für Studium und Praxis, 1991, S. 115. Mit Art. 3 VertragsG a.F. (Gesetz zu dem Übereinkommen der Vereinten Nationen vom 11. April 1980 über Verträge über den internationalen Warenkauf sowie zur Änderung des Gesetzes zu dem Übereinkommen vom 19. Mai 1989, BGBl. 1989 II 586) wurde argumentiert, dass der deutsche Gesetzgeber unter Vertragswidrigkeit ebenfalls nur Sachmängel verstehe, da in dieser internen Vorschrift für den Beginn der Verjährung nach § 477 BGB a.F. von Ansprüchen aus Art. 43 CISG in Bezug genommen wurde, vgl. Schlechtriem/Schlechtriem, a.a.O. (Fn. 5), Art. 3 VertagsG, Rn. 5. Dieser Gesichtspunkt kommt nicht mehr zum Tragen, da durch Umsetzung des Schuldrechtsmodernisierungsgesetzes (Art. 5 Abs.
30) die Bezugnahme auf Art. 39 CISG in Art. 3 VertragsG n.F. gestrichen wurde. Die Frage, ob Vertragswidrigkeit in Art. 3 VertragsG n.F. seinerseits für alle Leistungsstörungen gilt, kann im Rahmen dieses Beitrags nicht detailliert nachgegangen werden. Jedoch scheint die wörtliche Anlehnung an Art. 40 CISG dafür zu sprechen, dass der Gesetzgeber in erster Linie wiederum Sachmängel im Blick hatte. In Art. 10 Abs. 2 VerjÜbk (Übereinkommen über die Verjährung beim internationalen Warenkauf vom 14. Juni 1974 in der Fassung des Protokolls vom 11. April 1980; Deutschland ist diesem Übereinkommen bisher nicht beigetreten), werden Ansprüche wegen Rechtsmängel als Ansprüche aus Vertragswidrigkeit der Ware angesehen, siehe Schlechtriem/Müller-Chen, a.a.O. (Fn. 5), Art. 10 VerjÜbk, Rn. 4.

${ }^{24}$ Schlechtriem/Huber, a.a.O. (Fn. 5), Art. 46, Rn. 27; Art. 50, Rn. 8; Schlechtriem, a.a.O. (Fn. 18), Rn. 184, 202 (wenngleich a.A. rechtspolitisch wünschenswert); Hirner, a.a.O. (Fn. 15), S. 191ff., 214f.; Prager, a.a.O. (Fn. 16), S. 202f., 212, dessen Ansicht nach die Regelung allerdings nur als missglückt bezeichnet werden kann; Honsell/Schnyder/Straub, Kommentar zum UN-Kaufrecht, 1997, Art. 46, Rn. 6, 9; Art. 50, Rn. 11; Piltz, Internationales Kaufrecht: Das UN-Kaufrecht (Wiener Übereinkommen von 1980) in praxisorientierter Darstellung, 1993, § 5, Rn. 16, 149, 154, 304; Karollus, a.a.O. (Fn. 23), S. 115, 158; Tunón, The Actio Quanti Minoris and Sales of Goods between Mexico and the U.S.: An Analysis of the Remedy of Reduction of the Price in the UN Sales Convention, CISG Art. 50 and ist Civil Law Antecedents, http://www.cisg.law.pace.edu/ cisg/biblio/muria/html\#minoris, 4.2.3.; Piliounis, The Remedies of Specific Performance, Price Reduction and Additional Time (Nachfrist) under the CISG: Are these worthwhile changes or additions to English Sales Law?, 1999, http://www.cisg.law.pace.edu/cisg/biblio/piliounis.html\#6, 6) a) iii); so auch bereits Bergsten/Miller, The Remedy of Reduction of Price, 1979, http://www.cisg.law.pace.edu/cisg/biblio/bergsten.html, zu Art. 46 des New Yorker Entwurfs von 1978.

25 Sekretariatskommentar, O.R., Art. 39, Nr. 7, 8 (in: Honnold, a.a.O. (Fn. 14), S. 426); Art. 40, Nr. 12 (in: Honnold, a.a.O. (Fn. 14), S. 427). Versuche, deren Anwendungsbereich auf die Rechtsmängelhaftung zu erstrecken, blieben erfolglos, vgl. O.R., S. 118, Art. 46, Nr. 3 a.E. (Antrag Norwegens) (in: Honnold, a.a.O. (Fn. 14), S. 690); O.R., S. 118, Art. 46, Nr. 11 (Antrag zurückgenommen) (in: Honnold, a.a.O. (Fn. 14), S. 690) und O.R., S. 360f., Nr. 68-76 (in: Honnold, a.a.O. (Fn. 14), S. 581f.).

${ }^{26}$ Schlechtriem/Schwenzer, a.a.O. (Fn. 5), Art. 42, Rn. 26, die i.E. eine einheitliche Auslegung befürwortet.

27 Staudinger/Magnus, a.a.O. (Fn. 20), Art. 41, Rn. 26; Art. 50, Rn. 10; Soergel/Lüderitz/Schüßler-Langeheine, a.a.O. (Fn. 20), Art. 50, Rn. 2, die zwar erkennen, dass der Wortlaut gegen eine Minderung wegen Rechtsmangels spricht, i.E. aber eine entsprechende Anwendung des Art. 50 auf Rechtsmängel befürworten; Achilles, a.a.O. (Fn. 20), Art. 50, Rn. 2; Loewe, Internationales Kaufrecht, 1989, Art. 50, S. 72; Niggemann in: Hoyer/Posch, Das Einheitliche Wiener Kaufrecht, 1992, S. 106; Langenecker, a.a.O. (Fn. 16), S. 270f.

${ }^{28}$ Staudinger/Magnus, a.a.O. (Fn. 20), Art. 50, Rn. 9; Bianca/Bonell/ Will, Commentary on the International Sales Law, The 1980 Vienna Sales Convention, 1987, Art. 50, Nr. 3.4 (zweifelnd).

29 Enderlein/Maskow/Strohbach, a.a.O. (Fn. 20), Art. 50, Nr. 1; Biancal Bonell/Will, a.a.O. (Fn. 28), Art. 50, Nr. 3.4; Langenecker, a.a.O. (Fn. 16), S. 264.

${ }^{30}$ Honsell/Schnyder/Straub, a.a.O. (Fn. 24), Art. 45, Rn. 54.

${ }^{31}$ Honnold, Uniform Law for International Sales under the 1980 United Nations Convention, 3. Aufl., 1999, Rn. 313.1, der den Anwendungsbereich der Minderung auf „claims for non-conformity” beschränkt sieht und insbesondere auf die flexibleren und besser zutreffenden Bemessungsmöglichkeiten durch Schadensersatz hinweist.

32 Schlechtriem/Huber, a.a.O. (Fn. 5), Art. 44, Rn. 14; a.A. Langenecker, a.a.O. (Fn. 16), S. 264, 265.

33 Schlechtriem/Huber, a.a.O. (Fn. 5), Art. 44, Rn. 14. 


\section{Lösung des Sachproblems in Art. 82 Abs. 2 lit. c CISG}

In Bezug auf Art. 82 Abs. 2 lit. c CISG könnte entsprechend der einheitlichen Auslegung der Begriff der Vertragswidrigkeit wiederum so auszulegen sein, dass nur Sachmängel erfasst sind. Dies hätte zur Folge, dass dem Käufer seine Bösgläubigkeit nur im Falle eines Sachmangels schaden würde.

Oder muss die Frage anders entschieden werden, um zu einer sachgerechten Lösung in diesem Zusammenhang zu gelangen? Dann wäre die Prämisse der einheitlichen Auslegung widerlegt, was wiederum Auswirkungen auf die Auslegung an den anderen umstrittenen Gesetzesstellen zeitigen könnte.

Um die Frage beantworten zu können, ob in Art. 82 Abs. 2 lit. c CISG Sach- und Rechtsmängel gleich oder unterschiedlich zu behandeln sind, müssen zunächst deren Unterschiede vergegenwärtigt werden.

\section{Die Haftungsstandards der Art. 35, 41, 42 CISG}

Das Übereinkommen sieht verschiedene Standards für die Haftung wegen Sachmängel, Art. 35 CISG, allgemeiner Rechtsmängel, Art. 41 CISG, und Schutzrechtsmängel, Art. 42 CISG, vor.

Vergleicht man diese Normen miteinander, so fällt bezüglich des Haftungsausschlusses wegen Kenntnis des Käufers auf, dass die Art. 35 Abs. 3, Art. 42 Abs. 2 lit. a CISG denselben Wortlaut benutzen ${ }^{34}$. Demnach verliert der Käufer sachmangel- oder schutzrechtsbehafteter Ware seine Rechtsbehelfe, wenn er bei Vertragsschluss den Fehler kannte oder darüber nicht in Unkenntnis sein konnte.

Anders verhält es sich jedoch bei der allgemeinen Rechtsmängelhaftung nach Art. 41 CISG, bei der dem Käufer noch nicht einmal positive Kenntnis schadet ${ }^{35}$. Hier kann er seine Rechtsbehelfe nur durch Einwilligung ${ }^{36}$ verlieren.

\section{Vergleich der Haftungsstandards mit Art. 82 Abs. 2 lit. c CISG}

Während die Ausschlussregelungen der Art. 35, 41, 42 CISG die Haftung des Verkäufers insgesamt entfallen lassen, sperrt Art. 82 Abs. 2 lit. c CISG nur das eigentlich gem. Art. 49 CISG bestehende Recht zur Vertragsaufhebung. Nimmt man nun Art. 82 Abs. 2 lit. c CISG in die Vergleichsbetrachtung mit auf, so lässt sich für die Fälle der Sach- u. Schutzrechtsmängel Kongruenz feststellen, während bei allgemeinen Rechtsmängeln Differenzen sichtbar werden. Hinsichtlich einer Vertragsaufhebung schadet dem Käufer im Falle von Sach- oder Schutzrechtsmängeln bereits seine zurechenbare Unkenntnis, unabhängig davon, ob diese bereits im Zeitpunkt des Vertragsschlusses vorlag, Art. 35, 42 CISG, oder aber erst danach entstand, Art. 82 Abs. 2 lit. c CISG. Im Gegensatz dazu schadet dem Käufer von Ware, die mit allgemeinen Rechten Dritter belastet ist, bei Vertragsschluss zwar nur seine Einwilligung, Art. 41 CISG, in zeitlich späteren Stadien der Vertragsabwicklung würde aber auch hier seine zurechenbare Unkenntnis den Rechtsbehelf der Vertragsaufhebung sperren, Art. 82 Abs. 2 lit. c CISG. Dies würde allerdings voraussetzen, dass man unter Vertragswidrigkeit jede Form der Leistungsstörung verstünde. Das unsinnige Ergebnis dieser Einordnung wäre, dass demjenigen Käufer, der eine Minute vor Vertragsschluss Kenntnis erlangt, nicht aber demjeni- gen, der eine Minute nach Vertragsschluss davon erfährt, das Recht zu Vertragsaufhebung zustünde. Warum sollte man dem Käufer, der erst nach Vertragsschluss von einem Rechtsmangel Kenntnis erlangt, das Recht zur Vertragsaufhebung wegnehmen, obwohl man es ihm belassen würde, hätte seine Kenntnis bereits bei Vertragsschluss vorgelegen?

Aus diesem Grunde macht es im Fall der allgemeinen Rechtsmängelhaftung nach Art. 41 CISG keinen Sinn, dem Käufer das Recht zur Vertragsaufhebung gem. Art. 82 Abs. 2 lit. c CISG zu sperren.

Außerdem lässt sich das gegenteilige Ergebnis mit der ratio des Art. 82 Abs. 2 lit. c CISG, widersprüchliches Verhalten zu bestrafen, nicht in Einklang bringen. Dem Käufer, der trotz seiner Kenntnis rechtsmangelbehaftete Ware annimmt, schneidet man ja auch nicht die Rechtsbehelfe mit dem Einwand ab, er habe sich widersprüchlich verhalten, Art. 41 CISG. Im Gegenteil: Der Einwand des venire contra factum proprium ${ }^{37}$ wird, soweit es um die allgemeine Rechtsmängelhaftung nach Art. 41 CISG geht, implizit derogiert.

Im Zusammenhang mit Art. 82 Abs. 2 lit. c CISG ist es also sachlich geboten, den Begriff der Vertragswidrigkeit so zu verstehen, dass der Käufer, der nach Vertragsschluss von einem Sachmangel oder einem Schutzrechtsmangel Kenntnis erlangt und trotzdem die Ware weiterverkauft, nicht mehr den Vertrag aufheben darf. Hingegen schadet dem Käufer, der aufgrund eines allgemeinen Rechtsmangels aufheben will, seine Kenntnis nie, also unabhängig davon, ob sie bereits bei Vertragsschluss vorhanden oder erst danach entstanden war.

\section{Entstehungsgeschichte}

Die hier vertretene Auffassung lässt sich auch entstehungsgeschichtlich belegen ${ }^{38}$. Die maßgebliche Formulierung des Art. 82 Abs. 2 lit. c CISG basiert auf Art. 79 Abs. 2 lit. c EKG. Durch die Einbeziehung der Formulierung „have been sold in the normal course of business" wurde der Anwendungsbereich der Ausnahmen auf die Weiterveräußerung erstreckt ${ }^{39}$. Daneben wurde „or ought to have been discovered“ der positiven

34 Su, Die Rechtsmängelhaftung des Verkäufers nach UN-Kaufrecht und im chinesischen Recht, IPRax 1997, S. $284 \mathrm{ff.}, 287$.

35 Schlechtriem, Conformity of the Goods, Seller's Obligations under the United Nations Convention on Contracts for the International Sale of Goods, in: Galston/Smit (Hrsg.), International Sales, 1984, § 6.03, S. 6 31.

36 Der Streit, wann im einzelnen von einer Einwilligung gesprochen werden kann, ist unerheblich, da feststeht, dass Einwilligung jedenfalls mehr als bloße Kenntnis verlangt, vgl. Schlechtriem/Schwenzer, a.a.O. (Fn. 5), Art. 41, Rn. 17. Zwar kann sie auch konkludent erteilt werden (vgl. Schlechtriem, a.a.O. (Fn. 35), §6.03, S. 6-31), jedoch muss, um von einer Einwilligung durch vorbehaltlose Annahme sprechen zu können, beispielsweise der Käufer nach Anzeige des Schutzrechts eines Dritten durch den Verkäufer den Kaufpreis direkt an den Dritten zahlen.

37 Das Verbot des venire contra factum proprium wird allgemein als Grundsatz aus dem Gebot von Treu und Glauben nach Art. 7 Abs. 1 CISG hergeleitet, vgl. Schlechtriem/Ferrari, a.a.O. (Fn. 5), Art. 7, Rn. 50; Ferrari, Das Verhältnis zwischen den UNIDROIT-Grundsätzen und den allgemeinen Grundsätzen internationaler Einheitsprivatrechtskonventionen, JZ 1998, S. 12; Magnus, Die allgemeinen Grundsätze im UN-Kaufrecht, RabelsZ 59 (1995), S. 480f.

38 Vgl. zur allgemeinen Entstehungsgeschichte des Art. 82: Schlechtriem/ Leser/Hornung, a.a.O. (Fn. 5), Art. 82, Rn. 1 ff. 
Kenntnis hintangestellt, um einen objektiven Maßstab anlegen zu können ${ }^{40}$. Unabhängig von diesen Änderungen existierten aber bereits die Ausnahmen des Verbrauchs und der Verarbeitung, die ihrerseits nur einschlägig waren, wenn der Käufer die Vertragswidrigkeit nicht kannte, Art. 79 Abs. 2 lit. c EKG. Da es im EKG noch keine dem Art. 42 CISG entsprechende Regelung über Schutzrechtsmängelhaftung gab, unterschied Art. 79 Abs. 2 lit. c EKG durch Verwendung des Begriffs Vertragswidrigkeit nur zwischen Sach- und allgemeinen Rechtsmängeln, was - aus den oben zum CISG genannten Gründen - durchaus sachgerecht war. Da die gesetzlich ungeregelte Schutzrechtsmängelhaftung der allgemeinen Rechtsmängelhaftung zugeordnet wurde, fand Art. 52 EKG auf jene Anwendung ${ }^{41}$. Dies hatte zur Folge, dass dem Käufer auch bei Übernahme von Ware, auf der Schutzrechte Dritter lasteten, nur seine Einwilligung schadete $^{42}$. Demnach war es - aus den gleichen Gründen wie für die allgemeine Rechtsmängelhaftung - auch nicht sinnvoll, dem Käufer schutzrechtsbehafteter Ware das Recht zur Vertragsaufhebung wegen Art. 79 Abs. 2 lit. c EKG zu sperren. Die Notwendigkeit zur Abgrenzung der Schutzrechtsmängelhaftung entstand erst mit Einführung des jetzigen Art. $42 \mathrm{CISG}^{43}$, der sich inhaltlich nicht an der Haftung für allgemeine Rechtsmängel, sondern an der für Sachmängel orientiert ${ }^{44}$. Diese Ähnlichkeit der gesetzlichen Ausgestaltung wird durch die Gesetzestechnik verwischt, in dem die Schutzrechtsmängelhaftung zwar in einem eigenen Artikel nach, aber dennoch - entsprechend ihrem Phänomen als „Rechtsmangel“ - im Zusammenhang mit der allgemeinen Rechtsmängelhaftung unter dem Topos „Rechte und Ansprüche Dritter" behandelt wird.

\section{Ergebnis}

Als Ergebnis ist festzuhalten, dass, jedenfalls soweit es um Art. 82 Abs. 2 lit. c CISG geht, unter den Begriff der Vertragswidrigkeit sowohl Sachmängel als auch Schutzrechtsmängel fallen. Allgemeine Rechtsmängel sind hingegen nicht mitumfasst.

\section{IV. Übertragung des Ergebnisses auf die Rechtsbehelfe des Käufers}

Die im Rahmen des Art. 82 Abs. 2 lit. c CISG getroffene Abgrenzung erscheint zwingend, da nur so eine Abgrenzung vorgenommen werden kann, die sich in das System der Leistungsstörungen des Übereinkommens einfügt. Fraglich ist aber, ob die Unterteilung in Sach- und Schutzrechtsmängel einerseits und allgemeine Rechtsmängel andererseits auch in den Art. 46 Abs. 2, 3, Art. 50 CISG sinnvoll ist, sich also die hier gewonnenen Erkenntnisse auf den oben dargelegten Meinungsstreit übertragen lassen.

\section{1. Übertragung auf Art. 46 Abs. 2, 3 CISG}

Im Zusammenhang mit den Einschränkungen des Rechts auf Nacherfüllung gem. Art. 46 Abs. 2, 3 CISG greift die zu Art. 82 Abs. 2 lit. c CISG gefundene Lösung. Bei den Beratungen zum CISG wurde deutlich, dass im grenzüberschreitenden Warenverkehr eine Garantiehaftung des Verkäufers für weltweite Schutzrechtsfreiheit wegen des schutzrechtlichen Territorialitätsprinzips wenig Sinn machen würde ${ }^{45}$. Dies führte zu Haf- tungsbeschränkungen, so dass die Schutzrechtsmängelhaftung im Ergebnis stärker der Sachmängelhaftung als der allgemeinen Rechtsmängelhaftung ähnelt ${ }^{46}$. Wenn diese Strukturähnlichkeit für die Voraussetzungen einer Pflichtverletzung evident ist, so muss sich diese auch auf die Rechtsbehelfsebene durchschlagen. Ansonsten würde die gewollte Haftungsbeschränkung dadurch, dass dem Käufer Rechtsbehelfe eingeräumt werden, die den Verkäufer erheblich belasten ${ }^{47}$, umgangen werden. Deshalb ist im Ergebnis eine Übertragung auf Art. 46 Abs. 2, 3 CISG angezeigt.

39 Working Group, fifth session, document A/CN.9/87, YB V, S. 43, Art. 79, Nr. 146 (in: Honnold, a.a.O. (Fn. 14), S. 189). Nach der Entscheidung liest sich Art. 79 EKG, abgesehen von einigen unwesentlichen sprachlichen Veränderungen, genauso wie die jetzige Fassung des Art. 82 CISG, vgl. Working Group, fifth session, document A/CN.9/87, YB V, S. 43, Art. 79, Nr. 150 (in: Honnold, a.a.O. (Fn. 14), S. 189).

40 Schlechtriem/Leser/Hornung, a.a.O. (Fn. 5), Art. 82, Rn. 2; Song, Vertragsaufhebung und Rückabwicklung nach deutschem und koreanischem Recht und internationalem Einheitskaufrecht, Eine Analyse der Folgen des Untergangs oder einer Beeinträchtigung der Kaufsache vor oder nach Erklärung der Aufhebung, 1999, S. 237.

41 OLG Düsseldorf vom 20.01.1983, in: Schlechtriem/Magnus, Internationale Rechtsprechung zum EKG und EAG, 1987, Art. 76 EKG, Nr. 4, S. $400 \mathrm{ff}$. (Geschmacksmusterrecht als Rechtsmangel); Dölle/Neumayer, a.a.O. (Fn. 7), Art. 52, Rn. 17; Mertens/Rehbinder, Internationales Kaufrecht, Kommentar zu den Einheitlichen Kaufgesetzen, 1975, Art. 52, Rn. 5; Soergel/Lüderitz, Kommentar zum Bürgerlichen Gesetzbuch, Bd. 3, 1991, Art. 52 EKG, Rn. 2; Huber, Der UNCITRAL-Entwurf eines Übereinkommens über internationale Warenkaufverträge, RabelsZ 43 (1979), S. 502; vgl. bereits zum Römer Entwurf: Riese, RabelsZ 22 (1957), S. 74; zweifelnd Honnold, a.a.O. (Fn. 31), Rn. 268, der auch die Möglichkeit, insbesondere bei einer einstweiligen Verfügung (injunction), zur Behandlung als Sachmangel anspricht.

42 Eine Einwilligung wurde allerdings bereits in der vorbehaltlosen Annahme der Ware trotz positiver Kenntnis des Rechtsmangels gesehen, Dölle/ Neumayer, Kommentar zum Einheitlichen Kaufrecht, 1976, Art. 52 EKG, Rn. 12.

43 Der erste Entwurf einer speziellen Haftung für Schutzrechtsmängel wurde von einer Sonderarbeitsgruppe bei der zehnten Sitzung von UNCITRAL erarbeitet und stimmt mit der jetzigen Fassung des Art. 42 CISG im wesentlichen überein, vgl. YB VIII, S. 40f., Nr. 211ff. (in: Honnold, a.a.O. [Fn. 14], S. 333f.).

44 Schlechtriem/Schwenzer, a.a.O. (Fn. 5), Art. 42, Rn. 25; Schlechtriem, a.a.O. (Fn. 35), § 6.03, S. 6-33; Schlechtriem, Uniform Sales Law, The UNConvention on Contracts for the International Sale of Goods, 1986, S. 73, 74, der ausführt: "Nevertheless, it is apparent that this case (Art. 42 CISG) is regarded as a special category of breach of contract, closer to a lack of conformity than to a defect in title.” A.A. Prager, a.a.O. (Fn. 16), S. 224, der Art. 42 CISG der Rechtsmängelhaftung nach Art. 41 CISG zuordnet.

45 Sekretariatskommentar, O.R., S. 37, Art. 40, Nr. 4 (in: Honnold, a.a.O. [Fn. 14], S. 427); Schlechtriem/Schwenzer, a.a.O. (Fn. 5), Art. 42, Rn. 1.

46 Vgl. Fn. 44.

47 Ersatzlieferung bei Gattungsware, z.B. Lieferung unter einer anderen Marke, die kein Schutzrecht verletzt, oder Nachbesserung durch Ersatz der patentverletzenden durch schutzrechtsfreie Bestandteile belasten den Verkäufer im internationalen Handeln sehr, Schlechtriem/Schwenzer, a.a.O. (Fn. 5), Art. 42, Rn. 25; a.A. Langenecker, a.a.O. (Fn. 16), S. 268, demzufolge ein Rechtsmangel stets einen wesentlichen Vertragsbruch darstelle, so dass die als Zusatzerfordernis gedachte Wesentlichkeit in Art. 46 Abs. 2 CISG keine eigenständige Bedeutung erlange. 


\section{2. Übertragung auf Art. 50 CISG}

Die Übertragung der hier erarbeiteten Maßstäbe auf Art. 50 CISG ist gleichermaßen sachgerecht. Zum einen scheinen selbst die Befürworter der Minderung wegen „Rechtsmangels“ zumeist nicht den Fall eines allgemeinen Rechtsmangels, sondern den Fall eines Schutzrechtsmangels vor Augen zu haben ${ }^{48}$. Außerdem wurde in den Beratungen zum Übereinkommen der Schutzrechtsmangel selbstverständlich als eine den Tatbestand der Minderung erfüllende Leistungsstörung angesehen ${ }^{49}$. Gerade um die Abgrenzung zu den allgemeinen Rechtsmängeln zu ermöglichen, wurde am Wortlaut Vertragswidrigkeit festgehalten.

Zudem macht die Rechtsfolge der actio quantis minoris, eine Vertragsanpassung durch proportionale Herabsetzung des Kaufpreises, für den Fall des Verkaufs fremder Ware gem. Art. 41 CISG von vornherein keinen Sinn, da der Käufer die Ware nicht behalten möchte und deshalb den Vertrag aufheben wird $^{50}$. Freilich sind andere Fälle im Rahmen des Art. 41 CISG denkbar, in denen die Minderung eine sinnvolle Alternative böte. Da aber bei Rechtsmängeln nur selten eine Befreiung des Schadensersatzschuldners nach Art. 79 CISG vorliegt ${ }^{51}$, führt der mit der Minderung konkurrierende Schadensersatzanspruch aus Art. 45 Abs. 1 lit. b i.V.m. Art. 74 CISG zur Befriedigung des Käufers. Deshalb steht entsprechend dem Wortlaut des Art. 50 CISG dem Käufer von Ware, die mit einem allgemeinen Rechtsmangel belastet ist, das Recht zur Minderung nicht zu.

Dagegen stellt die Minderung im Falle von Schutzrechten Dritter in der Regel eine sinnvolle Reaktionsmöglichkeit des Käufers dar. Es handelt sich hierbei stets um Verwertungsrechte Dritter, deren wirtschaftliches Interesse vom Verkäufer bzw. auch vom Käufer durch Zahlung, z.B. einer Lizenzgebühr, befriedigt werden kann. Im Gegenzug erhält der Zahlende ein Nutzungsrecht, so dass die Vertragsabwicklung im Verhältnis Verkäufer/Käufer ungestört weiter vollzogen werden kann. Die Einbuße im Wert der Ware durch direkte Zahlung oder vorübergehende Handlungsbeschränkungen kann der Käufer dann im Wege der Minderung vom Kaufpreis abziehen. Im Ergebnis ist Art. 50 CISG als Rechtsbehelf nur im Falle eines Sach- oder Schutzrechtsmangels, nicht hingegen bei einem allgemeinen Rechtsmangel anwendbar.

\section{Ergebnis}

Im Ergebnis kann der Käufer sachmangel- oder schutzrechtsbehafteter Ware bei Vorliegen aller sonstigen Voraussetzungen zwar nur eingeschränkt Nacherfüllung verlangen und nur vor Bösgläubigkeit den Vertrag aufheben, dafür aber jederzeit den Kaufpreis mindern. Im Gegensatz dazu kann der Käufer von Ware, die mit einem allgemeinen Rechtsmangel belastet ist, zwar uneingeschränkt Nacherfüllung verlangen und trotz Bösgläubigkeit den Vertrag aufheben, jedoch nie die Minderung ausüben.

\section{Erstattung von Nutzungen und Schadensersatz als Korrektive der gestörten Rückabwicklung}

Abschließend ist zu erörtern, wie sich das Verhalten des Käufer steuern lässt, der in Kenntnis eines allgemeinen Rechtsmangels die Ware unter Wert und möglicherweise in der Absicht, den
Verkäufer zu schädigen, weiterveräußert. Nach bisheriger Auffassung wurde diese Fallkonstellation durch die Aufhebungssperre des Art. 82 Abs. 1 CISG geregelt. Dadurch, dass nach der hier vertretenen Auffassung der Käufer rechtsmängelbehafteter Ware trotz Kenntnis des Defekts die Ware weiterveräußern darf, ohne sein Aufhebungsrecht zu verlieren, können Situationen entstehen, in denen der Käufer weit unter Preis verkauft, beispielsweise die Ware verschleudert, danach den Vertrag aufhebt und vom Verkäufer Rückzahlung des vollen Kaufpreises zuzüglich Zinsen gem. Art. 81 Abs. 2 S. 1, Art. 84 Abs. 1 CISG fordert.

Das CISG entspricht nicht dem Konzept anderer moderner ${ }^{52}$ Leistungsstörungsordnungen ${ }^{53}$, die das Problem gestörter Rückabwicklungen nur noch als Haftungsproblem ansehen und dem Verkäufer, der die Ware nicht mehr zurückerhält, einen Wer-

48 Staudinger/Magnus, a.a.O. (Fn. 20), Art. 50, Rn. 10, mit folgendem Beispiel: „Die Ware ist mit Zeichen- oder Markenrechten belastet, die dem Käufer den weiteren Absatz verschließen; er kann und will sie aber innerbetrieblich nutzen. Wenn ein Schadensersatzanspruch hier ausnahmsweise an Art. 79 scheitert, ist der Käufer auf den Minderungsanspruch angewiesen.“; Herber/Czerwenka, a.a.O. (Fn. 10), Art. 50, Rn. 3, mit folgendem Beispiel: „Andererseits kann die Wahl dieses für den Käufer einfachsten Rechtsbehelfs sinnvoll sein, wenn er etwa eine nur regional durch gewerbliche Schutzrechte belastete Ware unter Aussparung dieser Region vertreibt $[\ldots]^{\prime \prime}$.

49 Schlechtriem sympathisierte zunächst mit dem Vorschlag der Norwegischen Delegation, der vorsah, den Wortlaut des Art. 46 (jetziger Art. 50 CISG) so zu erweitern, dass auch Rechtsmängel gem. Art. 39 (jetziger Art. 41 CISG) den Rechtsbehelf der Minderung auslösen könnten. Dennoch hielt er entgegen, dass Folgeprobleme entstünden. Zum einen sei die Minderung in manchen Fällen schlicht unbrauchbar. Zum anderen könne eine solche Änderung zu der Annahme führen, dass die Minderung im Falle des Art. 40 (jetziger Art. 42 CISG) nicht zulässig sei, vgl. O.R., S. 360, Nr. 74 (in: Honnold, a.a.O. [Fn. 14], S. 581).

50 Eine Minderung machte nur dann Sinn, wenn der Käufer in diesem Fall den Kaufpreis auf Null mindern könnte, was aber neben Bejahung der grundsätzlichen Frage, ob eine Minderung auf Null ohne Vorliegen der Voraussetzungen einer Vertragsaufhebung möglich ist, voraussetzte, dass die mangelhafte Ware objektiv wertlos (so Hirner, a.a.O. [Fn. 15], S. 177) ist. Letzteres kann aber ausschließlich bei sachmängelbehafteter Ware gegeben sein, da nur in diesem Fall auch der Verkäufer kein Interesse mehr an einer Rückabwicklung der Sachleistung hat. Im dem hier interessierenden Fall ist die Ware aber nur subjektiv wertlos, da dem Käufer nicht wirksam Eigentum verschafft wurde, jedoch der Verkäufer (und erst recht der Eigentümer) ein legitimes Interesse an der Rücklieferung der in physischer Hinsicht einwandfreien Ware hat. Im Ergebnis ist die Ware somit nicht objektiv wertlos. A.A. Wolff, a.a.O. (Fn. 10), S. 174, derzufolge gerade in diesen Fällen der Wert der Ware Null sei, also die Minderung einen adäquaten Rechtsbehelf des Käufers darstelle. Falls mit dieser Gegenauffassung argumentiert wird, dass die subjektive Wertlosigkeit genüge, so wäre dann aber eine Rückgewährpflicht des Käufers aus Treu und Glauben gem. Art. 7 Abs. 1 CISG herzuleiten.

51 Schlechtriem/Schwenzer, a.a.O. (Fn. 5), Art. 41, Rn. 21.

52 So Schlechtriem, a.a.O. (Fn. 18), Rn. 321.

53 So seit 1.1.2002 die interne Regelung des Rücktritts in $\S 346$ Abs. 2, 3 BGB; Art. 7.3.6 Abs. 1 S. 2 UNIDROIT-Principles of International Commercial Contracts: „If restitution in kind is not possible or appropriate allowance should be made in money whenever reasonable."; Art. 4.305-309 Principles of European Contract Law, vgl. Zimmermann, Konturen eines europäischen Vertragsrechts, JZ 1995, 477ff. (485); Art. 160 Abs. 3-7 Gandolfi-Entwurf (Code Europèen des Contrats), Vgl. Schwenzer, Rechtsbehelfe und Rückabwicklung im CISG, in den European und UNIDROIT 
tersatzanspruch gewähren ${ }^{54}$. Dieser Wertersatz schützt den Verkäufer zugleich vor dem hier behandelten Fall, in dem ihm als Mindestbetrag der objektive Wert der Ware zugeführt wird.

Unter Geltung des CISG schuldet der Käufer dem Verkäufer für den Fall, dass er die Ware nicht ganz zurückgeben kann, gem. Art. 84 Abs. 2 lit. b CISG, all benefits which he has derived from the goods“. Die deutsche Übersetzung spricht vom „Gegenwert aller Vorteile“, womit freilich nicht der Wert der Ware gemeint ist. Vielmehr muss der Käufer nur die konkret gezogenen Vorteile auskehren ${ }^{55}$. Wenn also der Käufer nur einen Schleuderpreis erzielt hat, so schuldet er zunächst einmal nur diesen. Zwar schadet dem Käufer von rechtsmängelbehafteter Ware seine Kenntnis nicht. Dennoch setzt der Tatbestand des Art. 82 Abs. 2 lit. c CISG voraus, dass der Käufer „im normalen Geschäftsverkehr“ verkauft hat. Da Schleuderverkäufe nicht dem normalen Geschäftsverkehr entsprechen ${ }^{56}$, fällt dieser Fall noch unter die Sperrwirkung der Regel des Art. 82 Abs. 1 CISG.

Demgegenüber ist der Fall des Käufers, der zwar nicht verschleudert, wohl aber unter Preis weiterveräußert, nicht mehr von der Sperrwirkung des Art. 82 Abs. 1 CISG erfasst. Ein Weiterverkauf in Schädigungsabsicht stellt jedenfalls immer die Verletzung einer vertraglichen Schutzpflicht ${ }^{57}$ dar, die auch im Rückabwicklungsverhältnis Bestand hat ${ }^{58}$ und zu Schadensersatz nach Maßgabe des Art. 74 CISG verpflichtet. Darüber hinaus ist der Käufer, der beabsichtigt, ein Zurückweisungsrecht, hier in Gestalt der Vertragsaufhebung, auszuüben, nach Art. 86 CISG zu besonderer Sorgfalt und zur Erhaltung der Ware verpflichtet ${ }^{59}$. Eine Verletzung dieser erhöhten Sorgfaltspflichten nach Art. 86 CISG zieht ebenfalls Schadensersatz nach sich ${ }^{60}$. Diese Lösung ist insbesondere für die Fälle relevant, in denen der Käufer zwar unter Preis, aber nicht in Schädigungsabsicht weiterverkauft bzw. dem Käufer eine eventuell vorhandene Schädigungsabsicht konkret nicht nachgewiesen werden kann ${ }^{61}$. Eine Befreiung des Schadenersatzschuldners gem. Art. 79 CISG kommt in diesen Fallkonstellationen nicht in Betracht.

Fraglich ist, in welchem Umfang Schadensersatz geleistet werden muss. Reicht es aus, wenn man dem Verkäufer den Wert der Ware sichert, oder muss ihm darüber hinaus auch der hypothetische Gewinn einer Weiterveräußerung im ordnungsgemäßen Geschäftsgang zustehen. Der Wert der Vorteile muss aus der objektiven Sicht eines verständnisvollen Dritten geschätzt werden. Dies wird regelmäßig den ursprünglichen Kaufpreis plus Nettogewinn ${ }^{62}$, den ein vernünftiger Geschäftspartner erwirtschaftet hätte, umfassen. Sonst würde das Rückabwicklungsgleichgewicht gestört, da der Käufer seinerseits gem. Art. 84 Abs. 1 CISG Zinsen auf den vollen Kaufpreis seit Zahlung erhält.

Es ist vorgeschlagen worden, dem Käufer sachmangel- oder schutzrechtsbehafteter Ware, der wegen Weiterverkaufs trotz Kenntnis von der Vertragsaufhebung gesperrt ist, mit Art. 51 CISG in der Weise zu helfen, dass er nur im Umfang des noch vorhandenen Teils der Ware aufheben $\mathrm{kann}^{63}$. M.E. ist dies nicht möglich, da eine solche Lösung die Pflicht zur Auskehrung des Surrogats nach Art. 84 Abs. 2 lit. b CISG umgeht, die aber gerade der „Preis“ dafür sein soll, dass der Käufer entgegen Art. 82 Abs. 1 CISG den Vertrag trotz Unmöglichkeit der Rückgabe der Ware aufheben darf.

\section{Resümee}

Im Zusammenhang mit der Vertragsaufhebungssperre des Art. 82 Abs. 2 lit. c CISG werden vom Begriff der Vertragswid- rigkeit sowohl Sachmängel als auch Schutzrechtsmängel erfasst. Folglich kann sich derjenige Käufer, dessen Ware mit einem allgemeinen Rechtsmangel i.S.d. Art. 41 CISG belastet ist, bei Vorliegen aller übrigen Anspruchsvoraussetzungen trotz Unmöglichkeit der Rückgabe der Ware und unbeschadet seiner Kenntnis des Rechtsmangels vom Vertrag lösen. Die daraus entstehenden Folgeprobleme einer gestörten Rückabwicklung aufgrund Vertragsaufhebung durch einen bösgläubigen Käufer lassen sich mit den allgemeinen Regeln über die Erstattung von Nutzungen und Schadensersatz uneingeschränkt steuern. Letztlich ist eine Übertragung der Differenzierung zwischen Sach- und Schutzrechtsmängeln einerseits und allgemeinen Rechtsmängeln andererseits auch auf die Käuferrechtsbehelfe Nacherfüllung und Minderung angezeigt. Somit ist im Ergebnis

Principles, im Gandolfi-Entwurf sowie im deutschen Schuldrechtsmodernisiserungsgesetz, in: Schlechtriem (Hrsg.), Wandlungen des Schuldrechts, 2002, S. 46ff.; Art. 6:265,269,271,272 Nieuw Burgerlijk Wetboek (Niederlande), vgl. Hartkamp, Mr. C. Asser's handleiding tot de beoefening van het Nederlands burgerlijk recht, Verbintenissenrecht, Teil II, 9. Aufl., 1993, Fn. 105, Rn. 534.

${ }^{54}$ Hornung, Die Rückabwicklung gescheiterter Verträge nach französischem, deutschem und nach Einheitsrecht: Gemeinsamkeiten, Unterschiede, Wechselwirkungen, 1998, S. 149, zu den UNIDROIT-Principles und den European Principles; Krebs, a.a.O. (Fn. 2), S. $92 \mathrm{ff}$.

55 Hornung, a.a.O. (Fn. 54), S. 152.

56 Song, a.a.O. (Fn. 40), S. 239; vgl. die Entscheidung des OLG Düsseldorf v. 10.2.1994, 6 U 119/93, CISG-online Nr. 115 (www.cisg-online.ch), in der allerdings offengelassen wurde, ob die Ausnahmen des Art. 82 Abs. 2 CISG wegen der "unter Preis verschleuderte[n] Restmenge“ oder der Kenntnis der Käuferin im Ergebnis nicht vorlagen.

${ }^{57}$ Anspruchsgrundlage ist Art. 61 Abs. 1 lit. b CISG.

58 Art. 81 Abs. 1 S. 1 CISG.

59 Otte, UN-Kaufrecht: Käuferrechte bei Weiterverarbeitung der Kaufsache bzw. unterlassener Untersuchung und Mängelanzeige, IPRax 1999, S. 352ff., 355; Schlechtriem/Bacher, a.a.O. (Fn. 5), Art. 86, Rn. 18; Art. 85, Rn. 12f.; Witz/Salger/Lorenz/Lorenz, International Einheitliches Kaufrecht, Praktiker-Kommentar und Vertragsgestaltung zum CISG, 2000, Vor Artt. 85-88, Rn. 2, demzufolge dieser Anspruch auch auf allgemeine Vertragstreuepflichten gem. Art. 7 Abs. 1 CISG gestützt werden könnte.

${ }^{60}$ Schlechtriem/Bacher, a.a.O. (Fn. 5), Vor Artt. 85-88, Rn. 4; Krebs, a.a.O. (Fn. 2), S. 130, 131.

${ }^{61}$ Die Beweislast trifft grundsätzlich denjenigen, der sich auf ihn begünstigende Umstände beruft. Dies ist hier der Verkäufer. Vgl. Schlechtriem/ Stoll, a.a.O. (Fn. 5), Art. 74, Rn. 47.

${ }^{62}$ Freilich kann der Käufer Kosten, die ihm infolge des Weiterverkaufs entstanden sind, in Abzug bringen.

${ }^{63}$ Heilmann, Mängelgewährleistung im UN-Kaufrecht, Voraussetzungen und Rechtsfolgen im Vergleich zum deutschen internen Kaufrecht und zu den Haager Einheitlichen Kaufgesetzen, 1994, S. 486; Freiburg, Das Recht auf Vertragsaufhebung im UN-Kaufrecht, Unter besonderer Berücksichtigung der Ausschlussgründe, 2001, S. 264, derzufolge sich der Verlust des Vertragsaufgebungsrechts in analoger Anwendung des Art. 51 Abs. 1 CISG grundsätzlich auf den betroffenen Teil beschränke; Schlechtriem/ Huber, a.a.O. (Fn. 5), Art. 49, Rn. 32, demzufolge wiederum dem Verkäufer bei unzumutbaren Nachteilen mit Treu und Glauben nach Art. 7 Abs. 1 CISG zu helfen wäre. Huber zitiert zu dieser Auffassung weiter BGH NJW 1982, 2730, 2732 und Honsell/Schnyder/Straub, a.a.O. (Fn. 24), Art. 49, Rn. 94, die aber beide nicht Fälle des Art. 82 CISG betreffen. Vielmehr kommt dort der Fall des zur Gesamtaufhebung berechtigten Käufers zur Sprache. Dieser könne freilich auch nur eine Teilaufhebung erklären. Das entspreche der ratio der Vertragsaufhebung als letztes Mittel. Voraussetzung sei die Zulässigkeit der Gesamtaufhebung. Im Fall von Art. 82 Abs. 1 CISG, 
der Begriff der Vertragswidrigkeit im CISG einheitlich so auszulegen, dass er Sachmängel i.S.d. Art. 35 CISG und Schutzrechtsmängel i.S.d. Art. 42 CISG erfasst ${ }^{64}$.

der eine Voraussetzung der Vertragsaufhebung normiert, ist die Gesamtaufhebung aber gerade nicht zulässig.

${ }^{64}$ Im Rahmen dieses Beitrags konnte nicht detailliert aufgezeigt werden, wie sich diese Auslegung auf die in Fn. 9 genannten anderen Geset- zesstellen auswirkt. Im Falle einer teilweisen Leistungsstörung kann der Käufer gem. Art. 51 CISG seine Rechtsbehelfe nur für den vertragswidrigen Teil ausüben. Nur bei dieser Vorschrift macht die hier vorgenommene Differenzierung ersichtlich keinen Sinn, da auch die Lieferung von Ware denkbar ist, die nur teilweise mit allgemeinen Rechten Dritter (Art. 41 CISG) belastet ist. In diesem Zusammenhang ist der Begriff der Vertragswidrigkeit untechnisch zu verstehen. An allen anderen Gesetzesstellen führt die hier vorgeschlagene Differenzierung zu sachgerechten Ergebnissen.

\section{UN-Kaufrecht}

\section{Art. 3I CISG}

Die Klausel „Lieferung frei Haus“ hat für sich genommen keinen handelsüblich eindeutigen Inhalt im Hinblick auf den Erfüllungsort. Fehlen im Vertrag weitere objektive Momente, die für ihre Auslegung maßgeblich sein könnten, ist bei nach dem CISG zu beurteilenden Verträgen von der Grundregel auszugehen, daß sich der Erfüllungsort am Sitz des Verkäufers befindet.

Deutschland: OLG Köln, 16.7.200I - I6 U 22/0I - rechtskräftig

(Vorinstanz: LG Aachen, Urteil vom 16.1.2001 - 10 O 196/ 00)

\section{Entscheidungsgründe:}

Das förmlich nicht zu beanstandende Rechtsmittel bleibt in der Sache erfolglos. Zu Recht hat das Landgericht die Klage als unzulässig abgewiesen, da die internationale Zuständigkeit des Landgerichts Aachen nicht gegeben ist. Bereits aufgrund der gesetzlichen Regelungen lässt sich eine solche Zuständigkeit für das Landgericht Aachen nicht feststellen, so dass es auf die Frage, ob aufgrund der von der Beklagten verwendeten allgemeinen Geschäftsbedingungen eine ausschließliche Zuständigkeit in Belgien begründet wird, nicht mehr ankommt.

Die internationale Zuständigkeit richtet sich im vorliegenden Fall nach Artikel 2, 3, 5 Nr. 1 EuGVÜ. Dessen Regelungen finden Anwendung, da sowohl Deutschland als auch Belgien Vertragsstaaten des EuGVÜ sind, Artikel 1 Abs. 1 EuGVÜ.

Da der Gerichtsstand des Wohnsitzes nach Artikel 2 Abs. 1 EuGVÜ hier nicht gewählt wurde - dieser läge am Sitz der Beklagten in Belgien -, kann sich die internationale Zuständigkeit nur gemäß Artikel 3 Abs. 1 EuGVÜ aus den Regelungen der folgenden Abschnitte 2 bis 6 des EuGVÜ ergeben. Indes liegen die Voraussetzungen für den hier allein in Betracht kommenden Gerichtsstand des Erfüllungsortes nach Artikel 5 Nr. 1 EuGVÜ nicht vor. Die Frage, wo der Erfüllungsort liegt, beurteilt sich nach dem Vertragsstatut des Vertrages, der der Klage zugrunde liegt. Dieses wiederum ist zu bestimmen nach dem interna- tionalen Privatrecht des angerufenen Gerichts, lex causae (vgl. Zöller/Geimer, ZPO, 22. Aufl., Artikel 5 EuGVÜ, Rn. 1; Geimer/ Schütze, Europäisches Zivilverfahrensrecht, Artikel 5, Rn. 65; Thomas/Putzo, ZPO, 22. Aufl., Artikel 5 EuGVÜ, Rn. 3). Maßgebend ist mithin deutsches Kollisionsrecht. Anknüpfungspunkt ist diejenige Verpflichtung, die den Gegenstand der Klage bildet. Bei einem Rechtsstreit über die Folgen einer Vertragsverletzung, wie er hier gegeben ist, kommt es für die Anwendung des Artikel 5 Nr. 1 EuGVÜ auf die Hauptpflicht an, deren Nichterfüllung geltend gemacht wird. Das ist hier die Lieferpflicht des Verkäufers (des Beklagten) (vgl. Zöller/Geimer, ZPO, a.a.O., Rn. 2; Geimer/Schütze, a.a.O., Artikel 5 Rn. 59).

Artikel 28 Abs. 1 und 2 EGBGB, der grundsätzlich das Vertragsstatut regelt, wird hier durch die Vorschriften des CISG verdrängt, das Anwendung findet, weil Deutschland und Belgien dem Übereinkommen der Vereinten Nationen über Verträge über den internationalen Warenkauf beigetreten sind (vgl. Gesetzessammlung Jayme/Hausmann, Anmerkung 1 zu CISG; im Verhältnis zu Belgien findet das Abkommen seit 1.11.1997 Anwendung). Das CISG selbst enthält keine ausdrückliche Regelung zum Erfüllungsort, sondern überlässt dessen Bestimmung in erster Linie den Vertragsvereinbarungen. Die hier von den Parteien herangezogene Vorschrift des Artikel 31 lit. a CISG, die den Ort der „Lieferung“ regelt, greift nur ein, wenn vertraglich ein Ort der Lieferung nicht bestimmt wurde (vgl. Schlechtriem/ Huber, Kommentar zum Einheitlichen UN-Kaufrecht, 3. Aufl., Artikel 31, Rn. 5).

Der im vorliegenden Fall zwischen den Parteien mündlich geschlossene Vertrag sieht ausdrücklich nichts für den Erfüllungsort vor. Die unstreitig getroffene Abrede „frei Hof" bedeutet lediglich, dass die Verkäuferin die Transportkosten übernimmt. Diese Klausel ist nämlich gemäß Artikel 8 Abs. 1 Abs. 2 CISG nach dem hypothetischen Parteiwillen, hilfsweise nach dem objektiven Erklärungsinhalt auszulegen. Ausreichende konkrete Anhaltspunkte zur Ermittlung eines hypothetischen Parteiwillens fehlen hier. Somit ist die Abrede „frei Hof" nach objektiven Kriterien zu verstehen. Die vergleichbare Abrede „frei Haus" enthält nach überwiegender Meinung keinen handelsüblich eindeutigen Inhalt; sie ist vielmehr je nach den Umständen des Einzelfalles auszulegen (so die Rechtssprechung, vgl. BGH NJW 84, 567; BGH NJW 97, 871; OLG Karlsruhe NJW-RR 93, 1371). Sonstige objektive Momente, die für die Auslegung im vorliegenden Fall maßgeblich sein könnten, sind nicht erkenn- 